\title{
Adaptive PID Control System Design Based on ASPR Property of Systems
}

\author{
Ikuro Mizumoto $^{1}$ and Zenta Iwai ${ }^{2}$ \\ ${ }^{1}$ Department of Mechanical Systems Engineering, Kumamoto University \\ ${ }^{2}$ Kumamoto Prefectural College of Technology
}

Japan

\section{Introduction}

PID control is one of the most common control schemes applied to many industrial processes and mechanical systems. Because, the PID can be tuned according to the experience of operators and can applied to uncertain system without a certain system's model. However in cases where there are some changes of system properties, it has been pointed out the difficulties of maintaining the desired control performance and stability during operation, and in some cases, it might be difficult to tune the PID parameters so as to satisfy the desired performance. Furthermore, the control plays a very important role in the improvement of production quality, accuracy and in reducing production costs. As a result a great deal of attention has been focused on automatic or self tuning of PID controllers (Astrom \& Hagglund, 1995), and in recent decades several kinds of auto-tuning PIDs including self-tuning schemes and adaptive control strategies have been proposed (Chang et al., 2003; Iwai et al., 2006; Kono et al., 2007; Ren et al., 2008; Tamura \& Ohmori, 2007; Yamamoto \& Shah, 2004; Yu et al., 2007). Unfortunately, most PID auto-tuning methods did not pay sufficient attention to the stability of the resulting PID control system and the tuned PID parameters did not guarantee the stability of the control system after any change of the systems.

In this Chapter, an adaptive PID control system design strategy based on the almost strictly positive real (ASPR) property for linear continuous-time systems will be presented. The adaptive PID scheme based on the ASPR property of the system can guarantee the asymptotic stability of the resulting PID control system and since the method presented in this chapter utilizes the characteristics of the ASPR-ness of the controlled system, the stability of the resulting adaptive control system can be guaranteed with certainty. The stability analysis will also be shown for ASPR systems. However, since most practical systems do not satisfy ASPR conditions, difficulties will appear in the practical application of the ASPR based adaptive PID control. In order to solve this problem, a robust parallel feedforward compensator (PFC) design method, which render the resulting augmented system with the PFC in parallel ASPR, will be provided.

The proposed adaptive PID control system can guarantee the stability, and by adjusting PID parameters adaptively, the method maintains a better control performance even if there are some changes of the system properties. In order to confirm the usefulness an effectiveness of 
the proposed adaptive PID design scheme for real world processes, the proposed method is applied to an unsaturated highly accelerated stress test system.

\section{Problem statement}

Consider a SISO continuous-time system with a relative degree of $\gamma$.

$$
\begin{aligned}
& \dot{x}(t)=A x(t)+\boldsymbol{b} u_{f}(t)+C_{d 1} \boldsymbol{w}_{d}(t) \\
& y(t)=\boldsymbol{c}^{T} \boldsymbol{x}(t)+\boldsymbol{d}_{1}^{T} \boldsymbol{w}_{d}(t)
\end{aligned}
$$

where $x(t) \in R^{n}$ is a state vector, $u(t)$ and $y(t) \in R$ are the input and the output of the system, respectively. $\boldsymbol{w}_{d}(t) \in R^{m \times 1}$ is a disturbance. The system (1) is not required to be stable and/or minimum-phase.

Suppose that the disturbance $w_{d}(t)$ and a reference signal $r(k)$ which the system output $y(t)$ is required to follow are generated by the following known exosystem:

$$
\begin{aligned}
\dot{\boldsymbol{w}}_{d}(t) & =A_{m} \boldsymbol{w}_{d}(t) \\
r(t) & =\boldsymbol{c}_{m}^{T} \boldsymbol{w}_{d}(t)
\end{aligned}
$$

with a characteristic polynomial,

$$
\operatorname{det}\left(\lambda I-A_{m}\right)=\lambda^{m}+\alpha_{m-1} \lambda^{m-1}+\cdots+\alpha_{1} \lambda+\alpha_{0}
$$

We assume that the exosystem is stable or neutrally stable. That is, all its eigenvalues are located on the left half-plane and/or the imaginary axis.

The objective is to design an adaptive PID controller so as to have the output $y(t)$ track the reference signal $r(t)$.

Remark 1: The exosystem is divided into two parts for the disturbance model and the reference signal. The part of reference signal is available so that $r(t)$ is known, but the part of disturbance is just a model of the disturbance and practical signal of the disturbance is not available, only the characteristic polynomial is known.

\subsection{Transformed system}

For the system (1) with a relative degree of $\gamma$, there exists a nonsingular variable transformation:

$$
\left[\begin{array}{l}
\boldsymbol{z}(t) \\
\boldsymbol{\eta}(t)
\end{array}\right]=\boldsymbol{\Phi} \boldsymbol{x}(t)
$$

such that the system (1) can be transformed into the form (Isidori, 1995):

$$
\begin{aligned}
& \dot{\boldsymbol{z}}(t)=A_{z} \boldsymbol{z}(t)+\boldsymbol{b}_{z} u_{f}(t)+C_{z} \boldsymbol{\eta}(t)+D_{d 1} \boldsymbol{w}_{d}(t) \\
& \dot{\boldsymbol{\eta}}(t)=Q_{\eta} \boldsymbol{\eta}(t)+\boldsymbol{c}_{\eta} z_{1}(t)+F_{d 1} \boldsymbol{w}_{d}(t) \\
& y(t)=[10 \cdot 0]\left[\begin{array}{l}
\boldsymbol{z}(t) \\
\boldsymbol{\eta}(t)
\end{array}\right]+\boldsymbol{d}_{1}^{T} \boldsymbol{w}_{d}(t)
\end{aligned}
$$

where

$$
\begin{array}{ll}
A_{z}=\left[\begin{array}{cc}
\mathbf{0} & \boldsymbol{I}_{\gamma-1 \times \gamma-1} \\
-a_{0} \cdots-a_{\gamma-1}
\end{array}\right] \quad \boldsymbol{b}_{z}=\left[\begin{array}{lll}
0 \cdots & \cdots & b_{z}
\end{array}\right]^{T} \quad C_{z}=\left[\begin{array}{c}
\mathbf{0} \\
\boldsymbol{c}_{z}^{T}
\end{array}\right] \\
\boldsymbol{c}_{\eta}=\left[\begin{array}{l}
\mathbf{0} \\
1
\end{array}\right] \quad b_{z}=c^{T} A^{\gamma-1} \boldsymbol{b} & D_{d 1} \in R^{\gamma \times m} \quad F_{d 1} \in R^{n-\gamma \times m}
\end{array}
$$




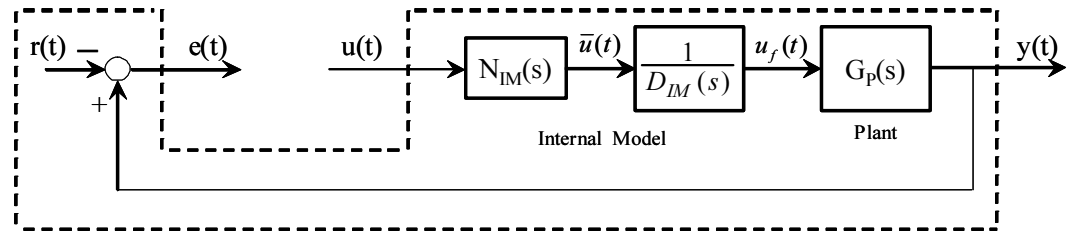

Fig. 1. Error System

\subsection{Error system with an internal model filter}

In order to alleviate the affect from disturbances and a PFC to be introduced later, we first consider the following internal model filter of the form:

$$
u_{f}(t)=G_{I M}(s)[u(t)]=\frac{N_{I M}(s)}{D_{I M}(s)}[u(t)]
$$

where

$$
D_{I M}(s)=\operatorname{det}\left(s I-A_{m}\right)
$$

and $N_{I M}(s)$ is any stable polynomial of order $m$ of the form:

$$
N_{I M}(s)=s^{m}+\beta_{m-1} s^{m-1}+\cdots+\beta_{1} s+\beta_{0}
$$

Defining the output following error by $e(t)=y(t)-r(t)$, consider the error system from $u(t)$ to $e(t)$ as shown Fig. 1 .

Define new variables $\boldsymbol{X}_{1}(t) \in R^{\gamma \times 1}, \boldsymbol{X}_{2}(t) \in R^{n-\gamma \times 1}$ as follows:

$$
\begin{aligned}
& \boldsymbol{X}_{1}(t)=\boldsymbol{z}^{(m)}(t)+\alpha_{m-1} \boldsymbol{z}^{(m-1)}(t)+\cdots+\alpha_{1} \dot{z}(t)+\alpha_{0} \boldsymbol{z}(t) \\
& \boldsymbol{X}_{2}(t)=\boldsymbol{\eta}^{(m)}(t)+\alpha_{m-1} \boldsymbol{\eta}^{(m-1)}(t)+\cdots+\alpha_{1} \dot{\boldsymbol{\eta}}(t)+\alpha_{0} \boldsymbol{\eta}(t)
\end{aligned}
$$

It follows from (2), (3), (5), (6) that

$$
\begin{aligned}
& \dot{\boldsymbol{X}}_{1}(t)=A_{z} \boldsymbol{X}_{1}(t)+\boldsymbol{b}_{z} \bar{u}(t)+C_{z} \boldsymbol{X}_{2}(t) \\
& \dot{\boldsymbol{X}}_{2}(t)=Q_{\eta} \boldsymbol{X}_{2}(t)+C_{\eta} \boldsymbol{X}_{1}(t)
\end{aligned}
$$

where

$$
\bar{u}(t)=u_{f}^{(m)}(t)+\alpha_{m-1} u_{f}^{(m-1)}(t)+\cdots+\alpha_{1} \dot{u}_{f}(t)+\alpha_{0} u_{f}(t)
$$

and

$$
C_{\eta}=\left[\begin{array}{ll}
0 & 0 \\
1 & 0
\end{array}\right]
$$

Next defining

$$
\boldsymbol{E}(t)=\left[e(t) \dot{e}(t) \cdots e^{(m-1)}(t)\right]^{T}
$$


we have from (2), (3) and (7) that

$$
\dot{\boldsymbol{E}}(t)=A_{E} \boldsymbol{E}(t)+C_{E} \boldsymbol{X}_{1}(t)
$$

where

$$
A_{E}=\left[\begin{array}{cc}
\mathbf{0} & \boldsymbol{I}_{m-1 \times m-1} \\
-\alpha_{0} & \cdots-\alpha_{m-1}
\end{array}\right] \in R^{m \times m}, \quad C_{E}=\left[\begin{array}{c}
\mathbf{0} \\
10 \cdots 0
\end{array}\right] \in R^{m \times \gamma}
$$

Thus, the error system can be represented by the following form without the term of disturbances:

$$
\begin{aligned}
\dot{\boldsymbol{E}}(t) & =A_{E} \boldsymbol{E}(t)+C_{E} \boldsymbol{X}_{1}(t) \\
\dot{\boldsymbol{X}}_{1}(t) & =A_{z} \boldsymbol{X}_{1}(t)+\boldsymbol{b}_{z} \bar{u}(t)+C_{z} \boldsymbol{X}_{2}(t) \\
\dot{\boldsymbol{X}}_{2}(t) & =Q_{\eta} \boldsymbol{X}_{2}(t)+C_{\eta} \boldsymbol{X}_{1}(t) \\
e(t) & =\left[\begin{array}{ll}
1 & 0
\end{array}\right] \boldsymbol{E}(t)
\end{aligned}
$$

This system with an input $\bar{u}(t)$ and the output $e(t)$ is of the order $n+m$ with a relative degree of $\gamma+m$. Since the error system has the relative degree of $\gamma+m$, the error system can be transformed into the following canonical form by an appropriate non-singular variable transformation.

$$
\begin{aligned}
\dot{z}_{z e}(t) & =A_{z e} z_{z e}(t)+\boldsymbol{b}_{z e} \bar{u}(t)+C_{z e} \boldsymbol{\eta}_{z e}(t) \\
\dot{\eta}_{z e}(t) & =Q_{z e} \eta_{z e}(t)+c_{\eta e} z_{e 1}(t) \\
e(t) & =z_{e 1}(t)
\end{aligned}
$$

with

$$
\begin{aligned}
& z_{z e}(t)=\left[\begin{array}{c}
z_{e 1}(t) \\
\vdots z_{e \gamma+m}(t)
\end{array}\right] \quad \eta_{z e}(t)=\left[\begin{array}{c}
z_{e \gamma+m+1}(t) \vdots \\
z_{e n+m}(t)
\end{array}\right]
\end{aligned}
$$

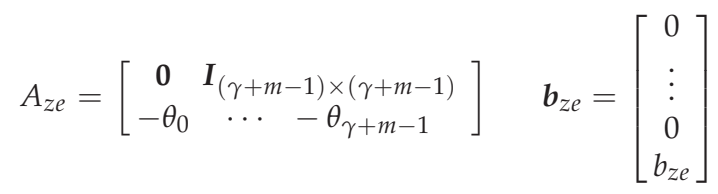

$$
\begin{aligned}
& C_{z e}=\left[\begin{array}{c}
0 \\
c_{z e}^{T}
\end{array}\right] \quad c_{\eta e}=\left[\begin{array}{l}
0 \\
1
\end{array}\right]
\end{aligned}
$$

and $\theta_{i}, b_{z e}$ and $c_{z e}$ are appropriate constants and vector. Further it follows from (9) that

$$
\begin{aligned}
& u_{f}^{(m)}(t)+\alpha_{m-1} u_{f}^{(m-1)}(t)+\cdots+\alpha_{1} \dot{u}_{f}(t)+\alpha_{0} u_{f}(t) \\
& =u^{(m)}(t)+\beta_{m-1} u^{(m-1)}(t)+\cdots+\beta_{1} \dot{u}(t)+\beta_{0} u(t) \\
& =\bar{u}(t)
\end{aligned}
$$


Defining

$$
\bar{z}_{I M}(t)=\left[u(t), \dot{u}(t), \cdots, u^{(m-1)}(t)\right]^{T}
$$

we have the following system representation from $\bar{u}(t)$ to $u(t)$.

$$
\begin{aligned}
\dot{\bar{z}}_{I M}(t) & =A_{I M} \bar{z}_{I M}(t)+\boldsymbol{b}_{I M} \bar{u}(t) \\
u(t) & =\overline{\boldsymbol{c}}_{I M}^{T} \overline{\boldsymbol{z}}_{I M}(t)
\end{aligned}
$$

where

$$
A_{I M}=\left[\begin{array}{cc}
\mathbf{0} & \boldsymbol{I}_{m-1 \times m-1} \\
-\beta_{0} & \cdots-\beta_{m-1}
\end{array}\right], \boldsymbol{b}_{I M}=\left[\begin{array}{l}
\mathbf{0} \\
1
\end{array}\right], \overline{\boldsymbol{c}}_{I M}^{T}=[10 \cdot 0]
$$

Consider the following variable transformation using the state variable $\bar{z}_{I M}(t)$ in (27).

$$
\begin{array}{r}
\xi_{k}(t)=-b_{z e} u^{(k-\gamma-1)}(t)+e^{(k-1)}(t)+\sum_{i=1}^{k-1} C_{\xi i} e^{(k-i-1)}(t) \\
(\gamma+1 \leq k \leq \gamma+m)
\end{array}
$$

where

$$
\begin{array}{cc}
C_{\xi i}=\left\{\begin{array}{cc}
-\Delta_{i}+\theta_{\gamma+m-i} & (1 \leq i \leq \gamma+m-1) \\
\theta_{0}-\sum_{j=1}^{m} \beta_{j-1} C_{\xi \gamma+j-1} & (i=\gamma+m)
\end{array}\right. \\
\Delta_{i}=\left\{\begin{array}{cc}
\beta_{m-1} & (i=1) \\
\beta_{m-i}+\sum_{j=1}^{m-1} \beta_{m-i+j} C_{\xi j} & (2 \leq i \leq m) \\
\sum_{j=1}^{m} \beta_{j-1} C_{\xi j-m+i-1} & (m+1 \leq i \leq \gamma+m-1)
\end{array}\right.
\end{array}
$$

Then it is easily to confirm that the error system (22) or (23) with $\bar{u}(t)$ as the input can be transformed into the following form with $u(t)$ as the input.

$$
\begin{aligned}
\dot{\boldsymbol{e}}(t) & =A_{e} \boldsymbol{e}(t)+\boldsymbol{b}_{e} u(t)+C_{e} \boldsymbol{\xi}(t) \\
\dot{\boldsymbol{\xi}}(t) & =A_{I M} \boldsymbol{\xi}(t)+B_{\xi} \boldsymbol{e}(t)+C_{\xi} \boldsymbol{\eta}_{z e}(t) \\
\dot{\boldsymbol{\eta}}_{z e}(t) & =Q_{z e} \boldsymbol{\eta}_{z e}(t)+\boldsymbol{c}_{\eta e} e(t) \\
e(t) & =[10] \boldsymbol{e}(t)
\end{aligned}
$$

where

$$
\boldsymbol{e}(t)=\left[e(t), \dot{e}(t), \cdots, e^{(\gamma-1)}(t)\right]^{T}, \boldsymbol{\xi}(t)=\left[\xi_{\gamma+1}(t), \cdots, \xi_{\gamma+m}(t)\right]^{T}
$$

and

$$
\begin{aligned}
& A_{e}=\left[\begin{array}{cc}
\mathbf{0} & \boldsymbol{I}_{\gamma-1 \times \gamma-1} \\
-C_{\xi \gamma} & \cdots-C_{\xi 1}
\end{array}\right], C_{e}=\left[\begin{array}{ll}
\mathbf{0} & 0 \\
1 & 0
\end{array}\right] \\
& \boldsymbol{b}_{e}=\left[0 \cdot 0 b_{z e}\right]^{T}, b_{z e}=c_{1}^{T} A_{1}^{\gamma+m-1} B_{1} \in R
\end{aligned}
$$




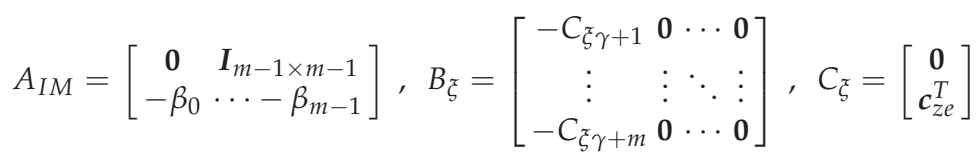

Note that this obtained error system with $u(t)$ as an input has relative degree of $\gamma$.

\section{Adaptive PID control system design}

Here we show an adaptive PID control system design scheme for the error system (32) based on system's ASPR properties.

\subsection{Almost Strictly Positive Realness (ASPR-ness)}

Let's consider the following $n$th order SISO system:

$$
\begin{aligned}
& \dot{x}(t)=A x(t)+\boldsymbol{b} u(t) \\
& y(t)=c^{T} x(t)
\end{aligned}
$$

where, $x(t) \in R^{n}$ is a state vector and $u(t), y(t) \in R$ are the input and the output, respectively. The ASPR-ness (almost strictly positive real-ness) of the system (36) is defined as follows:

Definition 1. (Bar-Kana \& Kaufman, 1985; Kaufman et al., 1997) The system (36) is called ASPR if there exists a static output feedback such that the resulting closed-loop system is strictly positive real (SPR). That is, system (36) is ASPR if there exists a control input with a feedback gain $\theta_{p}^{*}$,

$$
u(t)=-\theta_{p}^{*} y(t)+v(t), \quad \theta_{p}^{*}>0
$$

such that the resulting closed-loop system from $v(t)$ to $y(t)$ :

$$
\begin{gathered}
\dot{x}(t)=A_{c l} \boldsymbol{x}(t)+\boldsymbol{b} v(t) \\
y(t)=c^{T} \boldsymbol{x}(t) \\
A_{c l}=A-\theta_{p}^{*} \boldsymbol{b} c^{T}
\end{gathered}
$$

is strictly positive real (SPR).

The sufficient conditions for a system to be ASPR are given as follows (Kaufman et al., 1997):

(1) The relative degree of the system is 0 or 1.

(2) The system is minimum-phase.

(3) The high frequency gain of the system is positive.

Remark 2: The system (38) with the transfer function $G_{c}(s)=c^{T}\left(s I-A_{c l}\right)^{-1} \boldsymbol{b}$ is positive real if, for $\operatorname{Re}(s) \geq 0, \operatorname{Re} G_{\mathcal{C}}(s) \geq 0$, and it is SPR if , for some $\varepsilon>0, G_{c}(s-\varepsilon)$ is PR. Furthermore, if the system (38) is SPR, then there exist symmetric positive definite matrices $P$ and $Q$ such that the following Kalman-Yakubovich-Popov Lemma is satisfied.

$$
\begin{aligned}
A_{c l}^{T} P+P A_{c l} & =-Q \\
P \boldsymbol{b} & =c
\end{aligned}
$$




\subsection{Augmented ASPR controlled system design}

Since the error system (32) has a relative degree of $\gamma$ and also might be nonminimum-phase (If the system(1) is nonminimum-phase, then the error system (32) is nonminimum-phase), it is not ASPR. Here, how to make an augmented ASPR controlled system is first considered in order to design an adaptive PID controller based on the ASPR properties of the controlled system.

Let's consider the following parallel feedforward compensator (PFC) of order $n_{f}$ :

$$
\begin{aligned}
& \dot{x}_{f}(t)=A_{f} x_{f}(t)+b_{f} u(t) \\
& y_{f}(t)=c_{f}^{T} x_{f}(t)
\end{aligned}
$$

The augmented error system with the PFC (41) can be represented by

$$
\begin{aligned}
\dot{x}_{a}(t) & =A_{a} x_{a}(t)+\boldsymbol{b}_{a} u(t) \\
e_{a}(t) & =c_{a}^{T} x_{a}(t)=e(t)+y_{f}(t)
\end{aligned}
$$

where

$$
\begin{array}{rlrl}
\boldsymbol{x}_{a}(t)= & {\left[\boldsymbol{e}(t)^{T}, \boldsymbol{\xi}(t)^{T}, \boldsymbol{\eta}_{z e}(t)^{T}, \boldsymbol{x}_{f}(t)^{T}\right]^{T}} \\
A_{a}=\left[\begin{array}{cccc}
A_{e} & C_{e} & \mathbf{0} & \mathbf{0} \\
B_{\xi} & A_{I M} & C_{\xi} & \mathbf{0} \\
C & \mathbf{0} & Q_{z e} & \mathbf{0} \\
\mathbf{0} & \mathbf{0} & \mathbf{0} & A_{f}
\end{array}\right] & \boldsymbol{b}_{a}=\left[\begin{array}{c}
\boldsymbol{b}_{e} \\
\mathbf{0} \\
\mathbf{0} \\
\boldsymbol{b}_{f}
\end{array}\right] & \boldsymbol{c}_{a}=\left[\begin{array}{c}
1 \\
\mathbf{0} \\
\mathbf{0} \\
\boldsymbol{c}_{f}
\end{array}\right]
\end{array}
$$

Thus, if PFC is designed so as to render the resulting augmented system ASPR, we can consider the ASPR augmented system as a new controlled system.

\subsection{PFC design}

The PFC must render the resulting augmented system ASPR. Up to now several kinds of PFC design scheme which make the augmented system ASPR have been proposed (Iwai \& Mizumoto, 1994; Kaufman et al., 1997; Mizumoto \& Iwai, 1996). Here, we show a simple robust PFC design scheme based on an estimated or approximated model of the controlled system.

Let $G_{p}^{*}(s)$ be a given or roughly estimated approximated model of the controlled system (We suppose that any approximated model is available). A PFC, which renders the resulting augmented system ASPR, can be designed as follows:

$$
G_{P F C}(s)=\frac{1}{k}\left\{G_{A S P R}(s)-G_{p}^{*}(s)\right\}, k \geq 1
$$

where $G_{\text {ASPR }}(s)$ is a desired (or designed) ASPR model. In a general case, $k$ is designed as $k=1$, however, in order to expand the versatility of the PFC design, here we introduce a weight $k \geq 1$. The resulting augmented system can be represented by

$$
\begin{aligned}
G_{a}(s) & =G_{p}(s)+G_{P F C}(s) \\
& =G_{p}^{*}(s)+G_{P F C}(s)+G_{p}(s)-G_{p}^{*}(s) \\
& =\tilde{G}_{A S P R}(s)\{1+\Delta(s)\}
\end{aligned}
$$




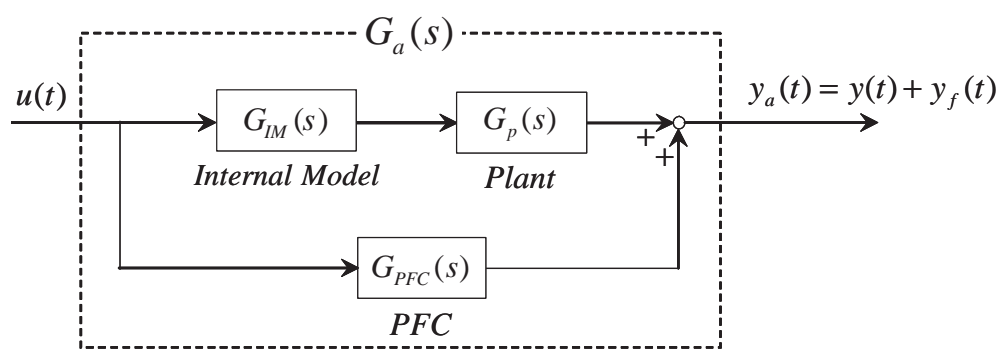

Fig. 2. Block diagram of the augmented system

where

$$
\begin{aligned}
\tilde{G}_{A S P R}(s) & =G_{p}^{*}(s)+G_{P F C}(s) \\
\Delta(s) & =\tilde{G}_{A S P R}(s)^{-1} \Delta G_{p}(s) \\
\Delta G_{p}(s) & =G_{p}(s)-G_{p}^{*}(s)
\end{aligned}
$$

$\Delta(s)$ represents an uncertain part of the augmented system.

The following lemma concerns the ASPR-ness of the resulting augmented system (45) (Mizumoto \& Iwai, 1996).

Lemma 1. The augmented system (45) is ASPR if

(1) $G_{A S P R}(s)$ is $A S P R$.

(2) $\Delta(s) \in R H_{\infty}$.

(3) $\|\Delta(s)\|_{\infty}<1$.

Where $\|\Delta(s)\|_{\infty}$ denote the $H_{\infty}$ norm of $\Delta(s)$ which is defined as $\|\Delta(s)\|_{\infty}=\sup _{s \in C_{+e}}|\Delta(s)|$.

Remark 3: Theoretically, one can select any ASPR model as $G_{A S P R}(s)$. However, performance of the control system may be influenced by the given ASPR model. For example, if the time constant of the given $G_{A S P R}(s)$ is small, one can attain fast tracking of the augmented system with small input. However, since the resulting PFC might have a large gain, the tracking of the practical output $y(t)$ has delay. One the centrally, if the time constant of $G_{A S P R}(s)$ is large, one can attain quick tracking for the practical output $y(t)$. However, large control input will be required (Minami et al., 2010).

The overall block diagram of the augmented system for the system with an internal model filter $G_{I M}(s)$ can be shown as in Fig. 2. Thus, introducing an internal model filter, the PFC must be designed for a system $G_{I M}(s) G_{p}(s)$. Unfortunately, in the case where $G_{I M}(s)$ is not stable the PFC design conditions given in Theorem 1 are not satisfied even if the controlled system $G_{p}(s)$ is originally stable. For such cases, the PFC can be designed according to the following procedure.

Step 1: Introduce a PFC as shown in Figure 3.

Step 2: Consider designing a PFC $G_{P F C}(z)$ so as to render the augmented system $G_{c}(s)=$ $G_{p}(s)+G_{P F C}(s)$ for the controlled system $G_{p}(z)$ ASPR. 


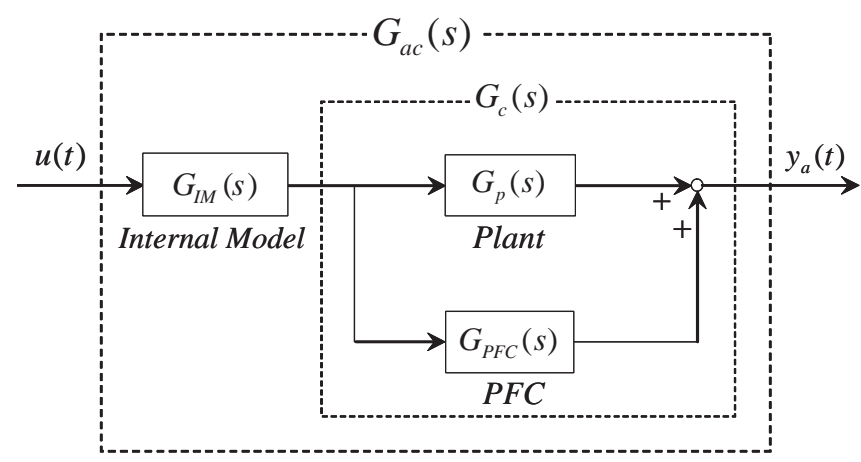

Fig. 3. Block diagram of a modified augmented system

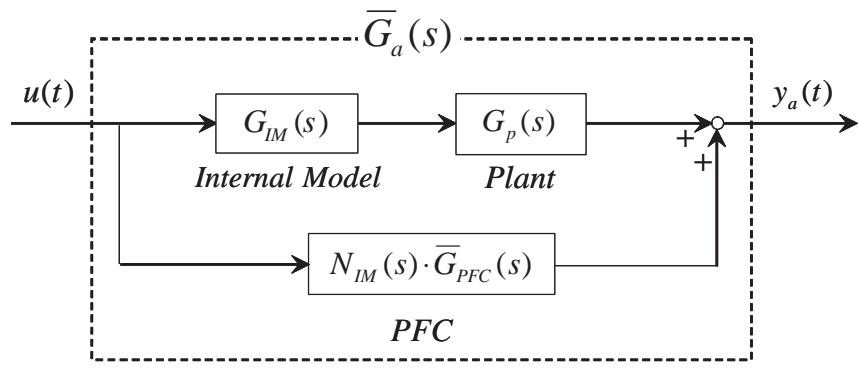

Fig. 4. Equivalent augmented system

Step 3: Design the desired ASPR model so that the obtained PFC $G_{P F C}(s)$ has $D_{I M}(s)$ as a part of the numerator. That is, the designed $G_{P F C}(z)$ must have a form of

$$
G_{P F C}(s)=D_{I M}(s) \cdot \bar{G}_{P F C}(s), \bar{G}_{P F C}(s)=\frac{\bar{N}_{P F C}(s)}{\bar{D}_{P F C}(s)}
$$

where $D_{I M}(s)$ and $\bar{D}_{P F C}(s)$ are coprime polynomials.

In this case, the obtained augmented system $G_{a c}(z)=G_{c}(s) G_{I M}(s)$ is ASPR since both $G_{c}(s)$ is ASPR and $G_{I M}(s)$ is ASPR with relative degree of 0 . Further, since the overall system given in Fig. 3 is equivalent to the system shown in Fig. 4, one can obtain an equivalent PFC that can render $G_{p}(s) G_{I M}(s)$ ASPR.

\subsection{Adaptive PID controller design}

For an ASPR controlled system with a PFC, let's consider an ideal PID control input given as follows:

$$
u^{*}(t)=-\tilde{\theta}_{p}^{*} e_{a}(t)-\tilde{\theta}_{i}^{*} w(t)-\tilde{\theta}_{d}^{*} \dot{e}_{a}(t)
$$

with

$$
\tilde{\theta}_{p}^{*}>0, \tilde{\theta}_{i}^{*}>0, \tilde{\theta}_{d}^{*}>0
$$

and

$$
\dot{w}(t)=e_{a}(t)-\sigma_{i} w(t), \sigma_{i}>0
$$


$w(t)$ is an pseudo-integral signal of $e_{a}(t)$ and $\tilde{\theta}_{p}^{*}$ is the ideal feedback gain which makes the resulting closed-loop of (42) SPR. That is, for the control system with $u^{*}(t)$ as the control input, considering a closed-loop system:

$$
\begin{aligned}
\dot{x}_{a}(t) & =A_{c} \boldsymbol{x}_{a}(t)+\boldsymbol{b}_{a} v(t) \\
e_{a}(t) & =c_{a}^{T} \boldsymbol{x}_{a}(t)
\end{aligned}
$$

where

$$
\begin{aligned}
A_{c} & =A_{a}-\tilde{\theta}_{p}^{*} \boldsymbol{b}_{a} \boldsymbol{c}_{a}^{T} \\
v(t) & =-\tilde{\theta}_{i}^{*} w(t)-\tilde{\theta}_{d}^{*} \dot{e}_{a}(t)
\end{aligned}
$$

the closed-loop system $\left(A_{c}, \boldsymbol{b}_{a}, \boldsymbol{c}_{a}\right)$ is SPR.

This means that the resulting control system with the input (48) will be stabilized by setting sufficiently large $\tilde{\theta}_{p}^{*}$ and any $\tilde{\theta}_{i}^{*}>0$ and $\tilde{\theta}_{d}^{*}>0$, which can be easily confirmed using the ASPR properties of the controlled system.

Unfortunately, however, since the controlled system is unknown, one can not design ideal PID gains. Therefore, we consider designing the PID controller adaptively by adaptively adjusting the PID parameters as follows:

$$
\begin{aligned}
u(t) & =-\tilde{\theta}_{p}(t) e_{a}(t)-\tilde{\theta}_{i}(t) w(t)-\tilde{\theta}_{d}(t) \dot{e}_{a}(t) \\
& =-\tilde{\boldsymbol{\theta}}(t)^{T} \tilde{\boldsymbol{z}}(t)
\end{aligned}
$$

where

$$
\begin{aligned}
\tilde{\boldsymbol{\theta}}(t)^{T} & =\left[\begin{array}{lll}
\tilde{\theta}_{p}(t) & \tilde{\theta}_{i}(t) & \tilde{\theta}_{d}(t)
\end{array}\right] \\
\tilde{\boldsymbol{z}}(t) & =\left[\begin{array}{lll}
e_{a}(t) & w(t) & \dot{e}_{a}(t)
\end{array}\right]^{T}
\end{aligned}
$$

and $\tilde{\boldsymbol{\theta}}(t)$ is adaptively adjusting by the following parameter adjusting law.

$$
\begin{aligned}
\dot{\tilde{\theta}}_{p}(t) & =\gamma_{p} e_{a}^{2}(t), \quad \gamma_{p}>0 \\
\dot{\tilde{\theta}}_{i}(t) & =\gamma_{i} w(t) e_{a}(t), \quad \gamma_{i}>0 \\
\dot{\tilde{\theta}}_{d}(t) & =\gamma_{d} \dot{e}_{a}(t) e_{a}(t), \quad \gamma_{d}>0
\end{aligned}
$$

The resulting closed-loop system can be represented as

$$
\begin{aligned}
\dot{x}_{a}(t) & =A_{c} \boldsymbol{x}_{a}(t)+\boldsymbol{b}_{a}\{\Delta u(t)+v(t)\} \\
e_{a}(t) & =\boldsymbol{c}_{a}^{T} \boldsymbol{x}_{a}(t)
\end{aligned}
$$

where

$$
\begin{aligned}
\Delta u(t) & =u(t)-u^{*}(t) \\
& =-\Delta \tilde{\boldsymbol{\theta}}(t)^{T} \tilde{\boldsymbol{z}}(t)
\end{aligned}
$$

with

$$
\Delta \tilde{\boldsymbol{\theta}}(t)=\left[\begin{array}{c}
\tilde{\theta}_{p}(t)-\tilde{\theta}_{p}^{*} \\
\tilde{\theta}_{i}(t)-\tilde{\theta}_{i}^{*} \\
\tilde{\theta}_{d}(t)-\tilde{\theta}_{d}^{*}
\end{array}\right]
$$




\subsection{Stability analysis}

Considering the ideal proportional gain $\tilde{\theta}_{p}^{*}$, the closed-loop system $\left(A_{c}, \boldsymbol{b}_{a}, \boldsymbol{c}_{a}\right)$ is SPR. Then there exist symmetric positive definite matrices $P=P^{T}>0, Q=Q^{T}>0$, such that the following Kalman-Yakubovich-Popov Lemma is satisfied

$$
\begin{aligned}
A_{c}^{T} P+P A_{c} & =-Q \\
P \boldsymbol{b}_{a} & =\boldsymbol{c}_{a}
\end{aligned}
$$

Now, consider the following positive definite function $V(t)$ :

$$
\begin{aligned}
V(t) & =V_{1}(t)+V_{2}(t)+V_{3}(t) \\
V_{1}(t) & =\boldsymbol{x}_{a}(t)^{T} P \boldsymbol{x}_{a}(t) \\
V_{2}(t) & =\tilde{\theta}_{i}^{*} w(t)^{2}+\tilde{\theta}_{d}^{*} e_{a}(t)^{2} \\
V_{3}(t) & =\Delta \tilde{\boldsymbol{\theta}}(t)^{T} \Gamma^{-1} \Delta \tilde{\boldsymbol{\theta}}(t)
\end{aligned}
$$

The time derivative of $V_{1}(t)$ can be expressed by

$$
\begin{aligned}
\dot{V}_{1}(t) & =\dot{x}_{a}(t)^{T} P x_{a}(t)+x_{a}(t)^{T} P \dot{x}_{a}(t) \\
& =x_{a}(t)^{T}\left(A_{c}^{T} P+P A_{c}\right) x_{a}(t)+2 b_{a}^{T} P x_{a}(t)\{\Delta u(t)+v(t)\} \\
& =-x_{a}(t)^{T} Q x_{a}(t)+2 e_{a}(t)\{\Delta u(t)+v(t)\}
\end{aligned}
$$

Further, the derivative of $V_{2}(t)$ is obtained as

$$
\begin{aligned}
\dot{V}_{2}(t) & =2 \tilde{\theta}_{i}^{*} w(t) \dot{w}(t)+2 \tilde{\theta}_{d}^{*} e_{a}(t) \dot{e}_{a}(t) \\
& =2 \tilde{\theta}_{i}^{*} w(t)\left\{e_{a}(t)-\sigma_{i} w(t)\right\}+2 \tilde{\theta}_{d}^{*} e_{a}(t) \dot{e}_{a}(t) \\
& =2 \tilde{\theta}_{i}^{*} w(t) e_{a}(t)+2 \tilde{\theta}_{d}^{*} \dot{e}_{a}(t) e_{a}(t)-2 \sigma_{i} \tilde{\theta}_{i}^{*} w(t)^{2} \\
& =-2 e_{a}(t) v(t)-2 \sigma_{i} \tilde{\theta}_{i}^{*} w(t)^{2}
\end{aligned}
$$

and the time derivative of $V_{3}(t)$ can be obtained by

$$
\begin{aligned}
\dot{V}_{3}(t) & =\Delta \dot{\tilde{\boldsymbol{\theta}}}(t)^{T} \Gamma^{-1} \Delta \tilde{\boldsymbol{\theta}}(t)+\Delta \tilde{\boldsymbol{\theta}}(t)^{T} \Gamma^{-1} \Delta \dot{\tilde{\boldsymbol{\theta}}}(t) \\
& =\frac{2}{\gamma_{p}} \Delta \tilde{\theta}_{p}(t) \Delta \dot{\tilde{\theta}}_{p}(t)+\frac{2}{\gamma_{i}} \Delta \tilde{\theta}_{i}(t) \Delta \dot{\tilde{\theta}}_{i}(t)+\frac{2}{\gamma_{d}} \Delta \tilde{\theta}_{d}(t) \Delta \dot{\tilde{\theta}}_{d}(t) \\
& =2 \Delta \tilde{\theta}_{p}(t) e_{a}(t)^{2}+2 \Delta \tilde{\theta}_{i}(t) w(t) e_{a}(t)+2 \Delta \tilde{\theta}_{d}(t) \dot{e}_{a}(t) e_{a}(t) \\
& =-2 \Delta u(t) e_{a}(t)
\end{aligned}
$$

Finally, we have

$$
\dot{V}(t)=-x_{a}(t)^{T} Q x_{a}(t) \leq 0
$$

and thus we can conclude that $\left\|x_{a}(t)\right\|$ is bounded and $L_{2}$ and all the signals in the control system are also bounded. Furthermore, form (42) and boundedness of all the signals in the control system, we have $\left\|\dot{x}_{a}(t)\right\| \in L_{\infty}$. Thus, using Barbalat's Lemma (Sastry \& Bodson, 1989), we obtain

$$
\lim _{t \rightarrow \infty} x_{a}(t)=0
$$


and then we can conclude that

$$
\lim _{t \rightarrow \infty} e(t)=0
$$

Remark 4: It should be noted that if there exist undesired disturbance and/or noise, one can not ensure the stability of the control system with the parameter adjusting law (55). In such case, one can design parameter adjusting laws as follows using $\sigma$-modification method:

$$
\begin{aligned}
\dot{\tilde{\theta}}_{p}(t) & =\gamma_{p} e_{a}^{2}(t)-\sigma_{P} \tilde{\theta}_{p}(t), \quad \gamma_{p}>0, \sigma_{P}>0 \\
\dot{\tilde{\theta}}_{i}(t) & =\gamma_{i} w(t) e_{a}(t)-\sigma_{I} \tilde{\theta}_{i}(t), \quad \gamma_{i}>0, \quad \sigma_{I}>0 \\
\dot{\tilde{\theta}}_{d}(t) & =\gamma_{d} \dot{e}_{a}(t) e_{a}(t)-\sigma_{D} \tilde{\theta}_{d}(t), \quad \gamma_{d}>0, \quad \sigma_{D}>0
\end{aligned}
$$

In this case, we only confirm the boundedness of all the signals in the control system.

Remark 5: If the exosystem (2) has unstable characteristic polynomial, then since $w_{d}(t)$ and/or $r(t)$ are not bounded, one cannot guarantee the boundedness of the signals in the control system, although it is attained that $\lim _{t \rightarrow \infty} e(t)=0$.

\section{Application to control of unsaturated highly accelerated stress test system}

\subsection{Unsaturated highly accelerated stress test system}

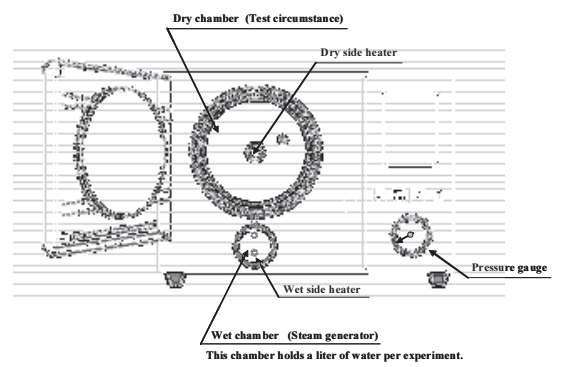

Fig. 5. Schematic view of the unsaturated HAST system

We consider to apply the ASPR based adaptive PID method to the control of an unsaturated HAST (Highly Accelerated Stress Test) system. Fig. 5 shows a schematic view of the unsaturated HAST system. In this system the temperature in the dry chamber has to raise quickly at a set point within 105.0 to 144.4 degree and must be kept at set point with $100 \%$ or $85 \%$ or $75 \% \mathrm{RH}$ (relative humidity). To this end, we control the temperature in the dry chamber and wet chamber by heaters setting in the chambers.

In the general unsaturated HAST system, the system is controlled by a conventional PID scheme with static PID gains. However, since the HAST system has highly nonlinearities and the system might be changed at higher temperature area upper than 100 degree and furthermore, the dry chamber and the wet chamber cause interference of temperatures each other, it was difficult to control this system by static PID. Fig. 6 shows the experimental result with a packaged PID under the control conditions of 120 degree in the dry chamber at $85 \% \mathrm{RH}$ (The result shows the performance of the HAST which is available in the market). The temperature in the dry chamber was oscillating and thus the relative humidity was also oscillated, and it takes long time to reach the set point stably. The requirement from the user is to attain a faster rising time and to maintain the steady state quickly. 


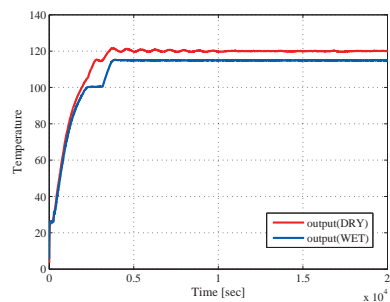

Fig. 6. Temperature in the dry chamber with a packaged PID: set point at 120 degree

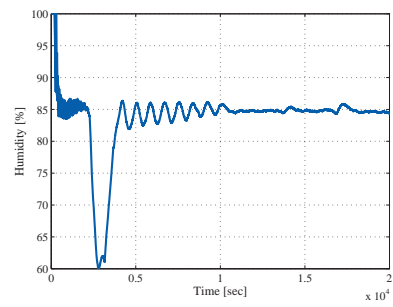

Fig. 7. Relative humidity with a packaged PID: $85 \%$ RH

\subsection{System's approximated model}

Using a step response under 100 degree, we first identify system models of dry chamber and wet chamber respectively (see Figs. 8).

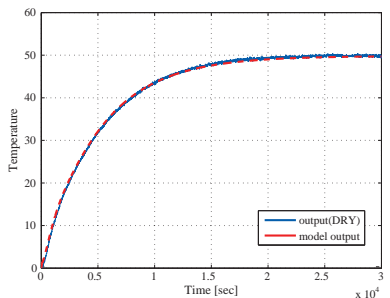

(a) Temperature in the dry chamber

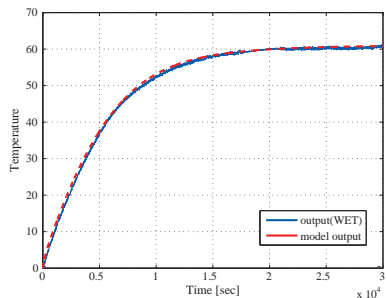

(b) Temperature in the wet chamber

Fig. 8. Step response

The identified models were obtained as follows by using Prony's Method (Iwai et al., 2005):

For dry chamber:

$$
\begin{gathered}
G_{P-D R Y}(s)=\frac{a_{1} s^{4}+b_{1} s^{3}+c_{1} s^{2}+d_{1} s+e_{1}}{s^{5}+f_{1} s^{4}+g_{1} s^{3}+h_{1} s^{2}+i_{1} s+j_{1}} \\
a_{1}=0.02146, b_{1}=0.000185, c_{1}=1.344 \times 10^{-6}, d_{1}=1.656 \times 10^{-9} \\
e_{1}=1.068 \times 10^{-12}, f_{1}=0.02373, g_{1}=0.0001138 \\
h_{1}=1.778 \times 10^{-7}, i_{1}=1.357 \times 10^{-10}, j_{1}=2.146 \times 10^{-14}
\end{gathered}
$$




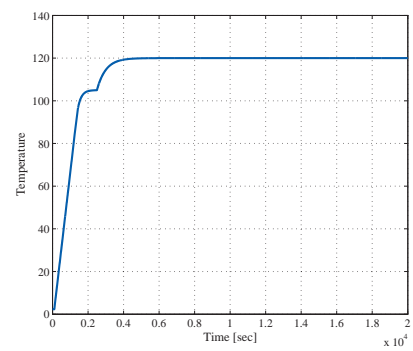

(a) Temperature in dry chamber

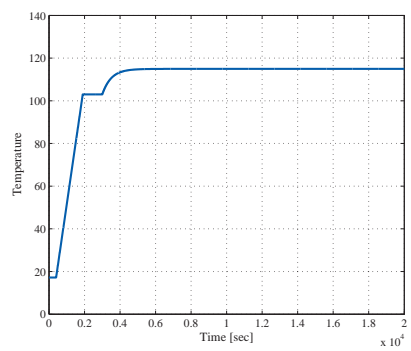

(b) Temperature in wet chamber

Fig. 9. Reference signals

\section{For wet chamber:}

$$
\begin{gathered}
G_{P-W E T}(s)=\frac{a_{2} s^{3}+b_{2} s^{2}+c_{2} s+d_{2}}{s^{4}+e_{2} s^{3}+f_{2} s^{2}+g_{2} s+h_{2}} \\
a_{2}=0.02122, b_{2}=7.078 \times 10^{-5}, c_{2}=3.906 \times 10^{-8} \\
d_{2}=9.488 \times 10^{-12}, e_{2}=0.006775, f_{2}=4.493 \times 10^{-6} \\
g_{2}=1.424 \times 10^{-9}, h_{2}=1.555 \times 10^{-13}
\end{gathered}
$$

It is noted that the HAST system is a two-input/two-output system so that we would have the following system representation.

$$
\left[\begin{array}{l}
y_{D R Y}(t) \\
y_{W E T}(t)
\end{array}\right]=\left[\begin{array}{ll}
G_{11}(s) & G_{12}(s) \\
G_{21}(s) & G_{22}(s)
\end{array}\right]\left[\begin{array}{l}
u_{D R Y}(t) \\
u_{W E T}(t)
\end{array}\right]
$$

For this system, we consider designing a decentralized adaptive PID controller to each control input $u_{D R Y}(t)$ and $u_{\text {WET }}(t)$. Therefore, in order to design PFCs for each subsystem, we only identified subsystems $G_{11}(s)=G_{P-D R Y}(s)$ and $G_{22}(s)=G_{P-W E T}(s)$.

\subsection{Control system design}

The control objective is to have outputs $y_{D R Y}(t)$ and $y_{W E T}(t)$, which are temperatures in the dry chamber and the wet chamber respectively, track a desired reference signal to attain a desired temperature in dry chamber and desired relative humidity. For example, if one would like to attain a test condition with the temperature in dry chamber of 120 degree with $85 \% \mathrm{RH}$, the reference signals shown in Fig. 9 will be set.

In order to attain control objective, we first design internal model filters as follows:

$$
G_{I M-D R Y}(s)=\frac{100 s+1}{s}, G_{I M-W E T}(s)=\frac{170 s+1}{s}
$$

Further, for each controlled subsystem with the internal models, we set desired ASPR models as follows in order to design PFCs for each subsystems.

$$
G_{A S P R-D R Y}(s)=\frac{49.8}{250 s+1}, G_{A S P R-W E T}(s)=\frac{61.0}{100 s+1}
$$


Then the PFCs were designed according to the model-based PFC design scheme given in (44) using obtained approximated model $G_{P-D R Y}(s)$ and $G_{P-W E T}(s)$ as follows:

$$
\begin{aligned}
& G_{P F C-D R Y}(s)=\frac{1}{k_{D R Y}}\left\{G_{A S P R-D R Y}(s)-G_{P-D R Y}(s)\right\}, k_{D R Y}=100 \\
& G_{P F C-W E T}(s)=\frac{1}{k_{W E T}}\left\{G_{A S P R-W E T}(s)-G_{P-W E T}(s)\right\}, k_{W E T}=170
\end{aligned}
$$

For the obtained ASPR augmented subsystems with PFCs, the adaptive PID controllers are designed as in (53) with parameter adjusting laws given in (71). The designed parameters in (71) are given as follows:

$$
\begin{gathered}
\Gamma_{D R Y}=\Gamma_{W E T}=\operatorname{diag}\left[\gamma_{d}, \gamma_{i}, \gamma_{d}\right]=\operatorname{diag}\left[1 \times 10^{-2}, 1 \times 10^{-5}, 1 \times 10^{-8}\right] \\
\sigma_{D}=\sigma_{I}=\sigma_{D}==1.0 \times 10^{-10} \\
\sigma_{i}=0
\end{gathered}
$$

\subsection{Experimental results}

We performed the following 4 types experiments.

(1) Quickly raise the temperature up to 120 degree and keep the relative humidity at $85 \%$ $\mathrm{RH}$.

(2) Quickly raise the temperature up to 130 degree and keep the relative humidity at $85 \%$ $\mathrm{RH}$.

(3) Quickly raise the temperature up to 121 degree and keep the relative humidity at $100 \%$ $\mathrm{RH}$.

(4) Quickly raise the temperature up to 120 degree and change the temperature to 130 and again 120 with keeping the relative humidity at $85 \% \mathrm{RH}$.

Figs. 10 to 13 show the results for Experiment (1). Fig. 10 shows the temperature in the dry and wet chambers and the relative humidity. It can be seen that temperatures quickly reached to the desired values and the relative humidity was kept at set value. Fig. 11 shows the results with the given reference signal. Both temperatures in dry and wet chamber track the reference signal well. Fig. 12 are control inputs and Fig. 13 shows adaptively adjusted PID parameters. Figs. 14 to 17 show the resilts for Experiment (2), Figs. 18 to 21 show the resilts for Experiment (3) and Figs. 22 to 25 show the resilts for Experiment (4). All cases attain satisfactory performance.

\section{Conclusion}

In this Chapter, an ASPR based adaptive PID control system design strategy for linear continuous-time systems was presented. The adaptive PID scheme based on the ASPR property of the system can guarantee the asymptotic stability of the resulting PID control system and since the method presented in this chapter utilizes the characteristics of the ASPR-ness of the controlled system, the stability of the resulting adaptive control system can be guaranteed with certainty. Furthermore, by adjusting PID parameters adaptively, the method maintains a better control performance even if there are some changes of the system properties. In order to illustrate the effectiveness of the presented adaptive PID design scheme for real world processes, the method was applied to control of an unsaturated highly accelerated stress test system. 


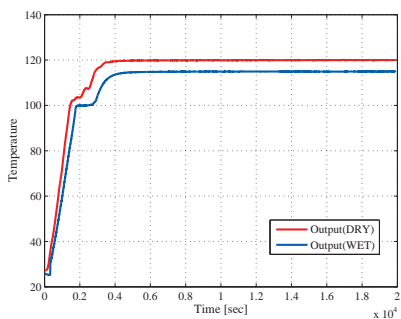

(a) Temperatures in the dry and wet chambers

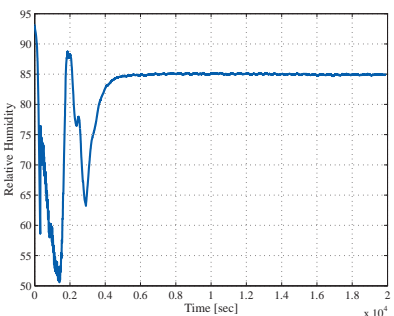

(b)Relative humidity

Fig. 10. Experimental results of outputs: 120 degree and $85 \% \mathrm{RH}$

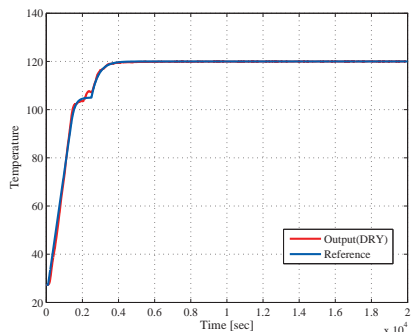

(a) Dry chamber

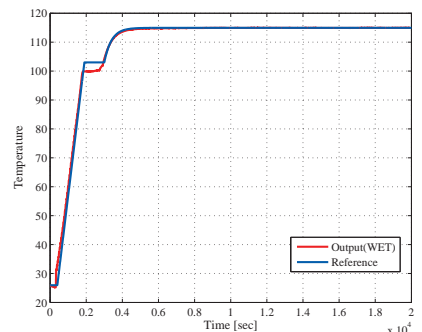

(b) Wet chamber

Fig. 11. Comparison between Output and Reference signal: 120 degree and $85 \% \mathrm{RH}$

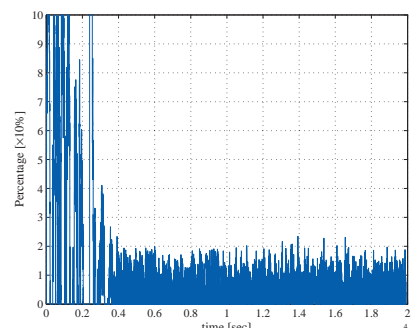

(a) Dry chamber

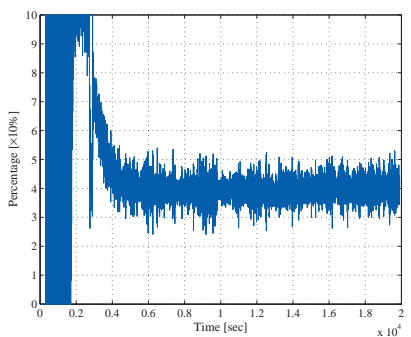

(b) Wet chamber

Fig. 12. Control Input: 120 degree and $85 \%$ RH
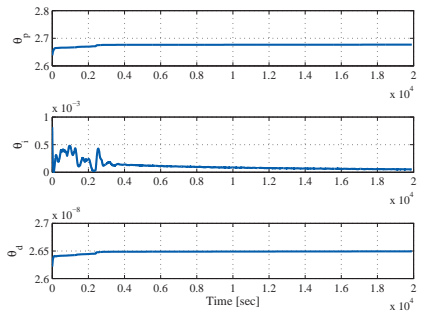

(a) Dry chamber
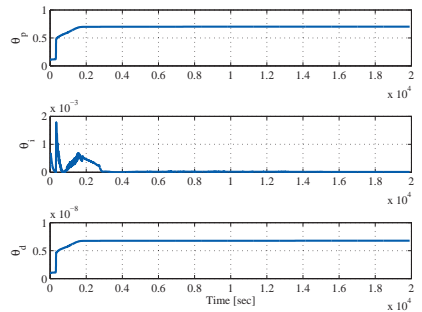

(b) Wet chamber

Fig. 13. Adaptively adjusted PID gains: 120 degree and $85 \% \mathrm{RH}$ 


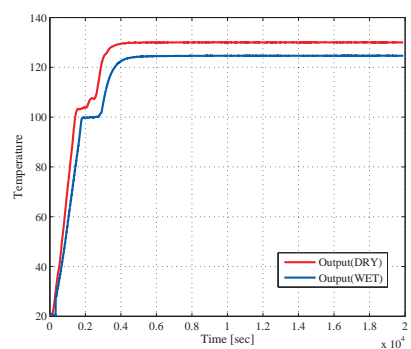

(a) Temperatures in the dry and wet chambers

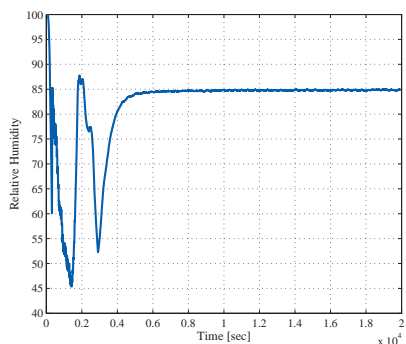

(b)Relative humidity

Fig. 14. Experimental results of outputs: 130 degree and $85 \% \mathrm{RH}$

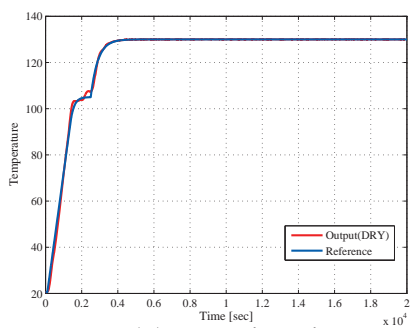

(a) Dry chamber

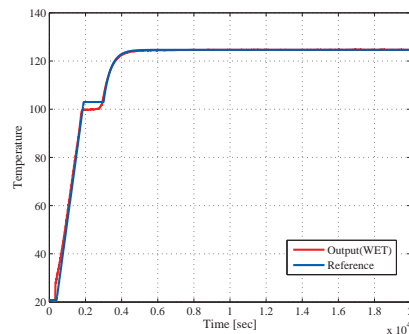

(b) Wet chamber

Fig. 15. Comparison between Output and Reference signal: 130 degree and $85 \%$ RH

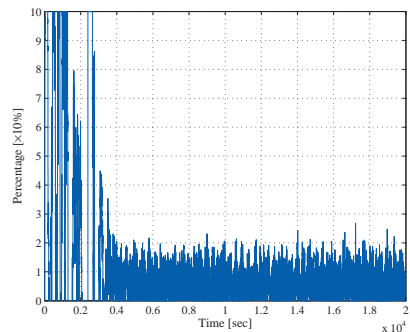

(a) Dry chamber

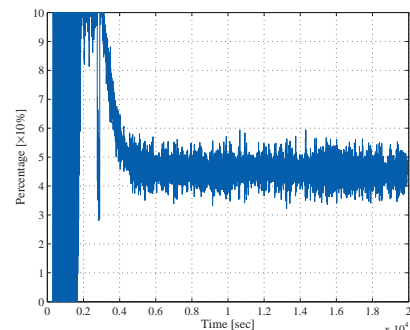

(b) Wet chamber

Fig. 16. Control Input: 130 degree and $85 \%$ RH

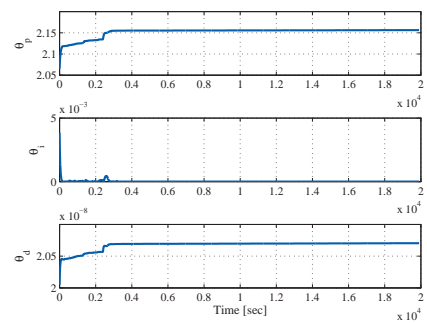

(a) Dry chamber

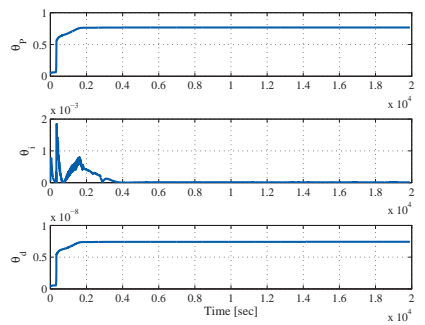

(b) Wet chamber

Fig. 17. Adaptively adjusted PID gains: 130 degree and $85 \% \mathrm{RH}$ 


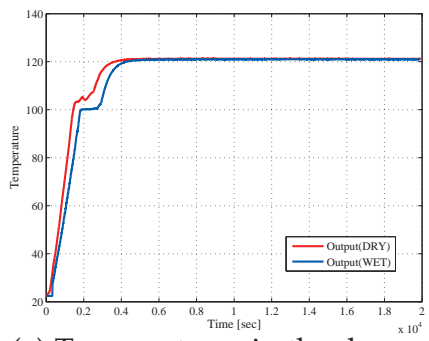

(a) Temperatures in the dry and wet chambers

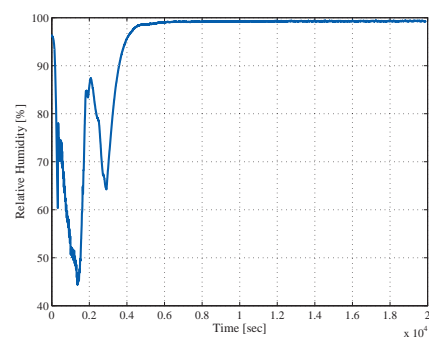

(b)Relative humidity

Fig. 18. Experimental results of outputs: 121 degree and $100 \% \mathrm{RH}$

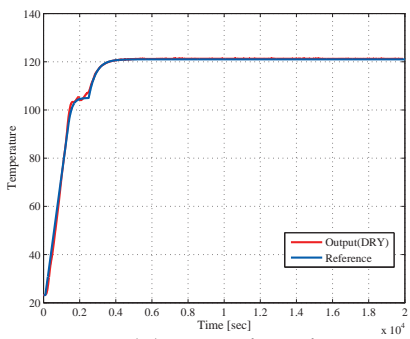

(a) Dry chamber

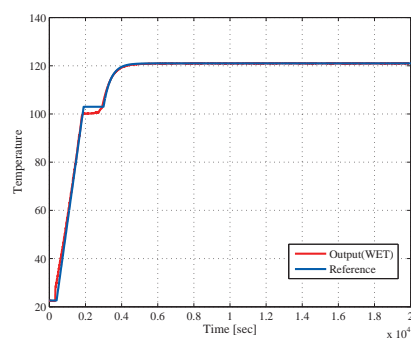

(b) Wet chamber

Fig. 19. Comparison between Output and Reference signal: 121 degree and $100 \%$ RH

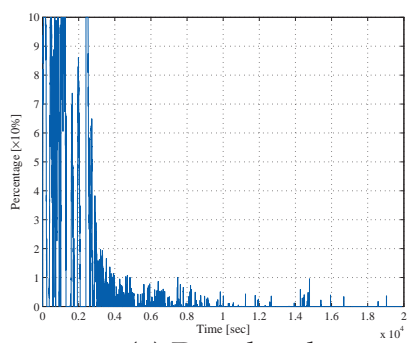

(a) Dry chamber

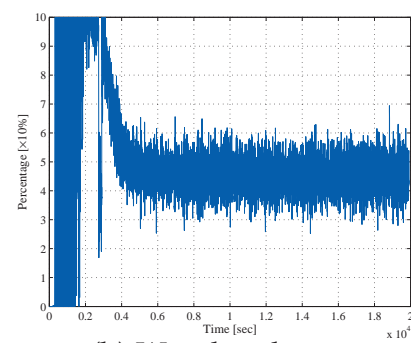

(b) Wet chamber

Fig. 20. Control Input: 121 degree and $100 \%$ RH
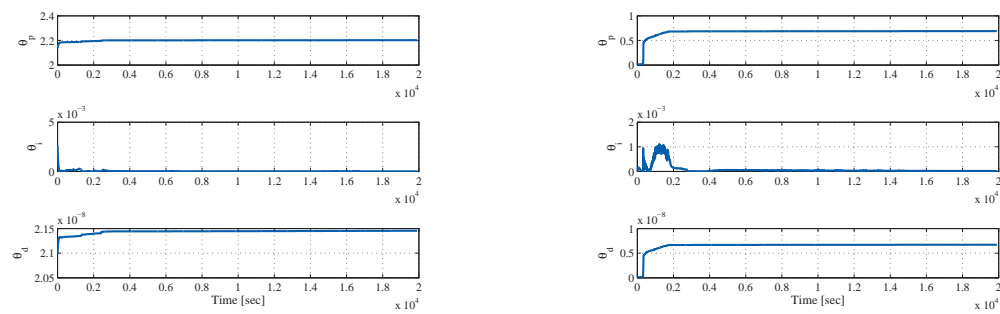

(a) Dry chamber

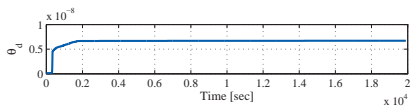

(b) Wet chamber

Fig. 21. Adaptively adjusted PID gains: 121 degree and $100 \% \mathrm{RH}$ 


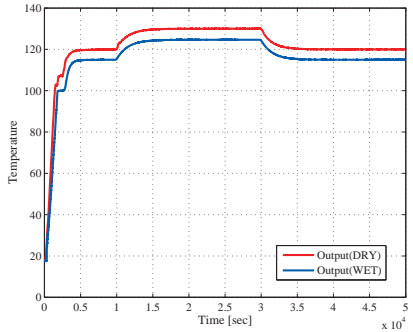

(a) Temperatures in the dry and wet chambers

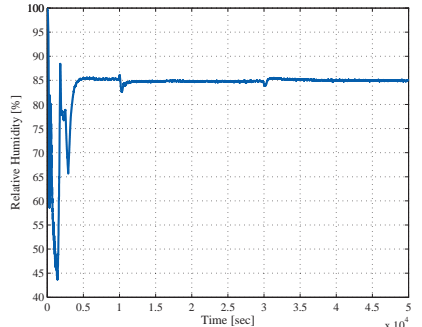

(b)Relative humidity

Fig. 22. Experimental results of outputs: $120 \rightarrow 130 \rightarrow 120$ degree with $85 \%$ RH

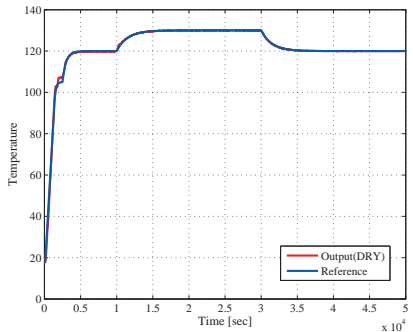

(a) Dry chamber

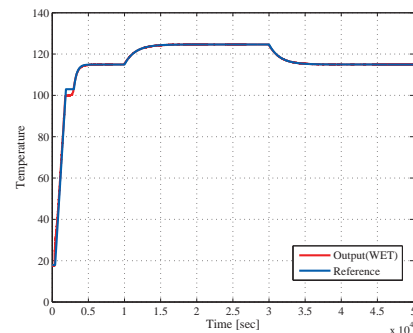

(b) Wet chamber

Fig. 23. Comparison between Output and Reference signal: $120 \rightarrow 130 \rightarrow 120$ degree with 85 $\% \mathrm{RH}$

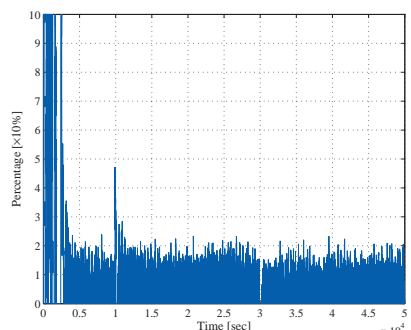

(a) Dry chamber

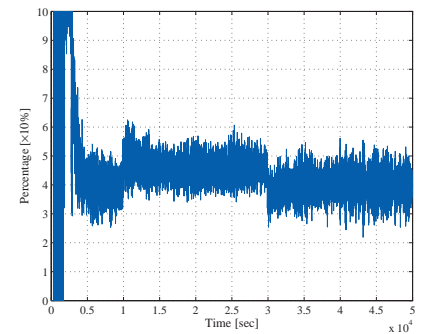

(b) Wet chamber

Fig. 24. Control Input: $120 \rightarrow 130 \rightarrow 120$ degree with $85 \%$ RH

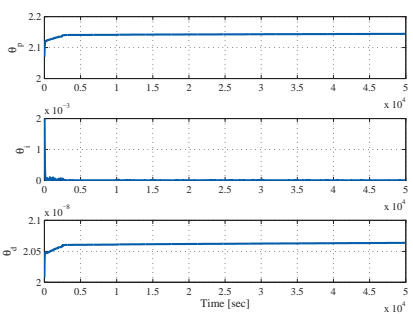

(a) Dry chamber

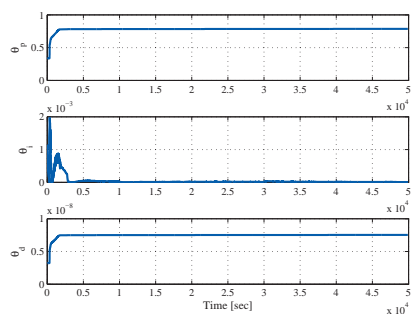

(b) Wet chamber

Fig. 25. Adaptively adjusted PID gains: $120 \rightarrow 130 \rightarrow 120$ degree with $85 \% \mathrm{RH}$ 


\section{References}

Astrom, K. \& Hagglund, T. (1995). Pid control, theory, design and tuning, Instrument Society of America, USA, second Ed.

Bar-Kana \& Kaufman, H. (1985). Global stability and performance of a simplified adaptive algorithm, International Journal of Control 42(6): 1491-1505.

Chang, W.-D., Hwang, R.-C. \& Hsieh, J.-G. (2003). A multivariable on-line adaptive pid controller using auto-tuning neurons, Engineering Application of Artificial Intelligence 16: 57-63.

Isidori, A. (1995). Nonlinear control systems, Springer-Verlag, third Ed.

Iwai, Z. \& Mizumoto, I. (1994). Realization of simple adaptive control by using parallel feedforward compensator, Int. J. of Control 59(6): 1543-1565.

Iwai, Z., Mizumoto, I., Liu, L., Shah, S. \& Jiang, H. (2006). Adaptive stable pid controller with parallel feedforward compensator, Proc. of 9th Int. Conf. on Control, Automation, Robotics and Vision, Singapore pp. 1253-1258.

Iwai, Z., Mizumoto, I., Nagata, M., Kumon, M. \& Kubo, Y. (2005). Accuracy of identification and control performance in 3 parameter process model approximations (identification by prony's method and examination through model-driven pid control system design), Trans. of the Japan Society of Mechanical Engineers (Ser. C) 71(702): 589-596.

Kaufman, H., Barkana, I. \& Sobel, K. (1997). Direct Adaptive Control Algorithms, 2nd edn, Springer.

Kono, T., Yamamoto, T., Hinamoto, T. \& Shah, S. (2007). Design of a data-driven performance-adaptive pid controller, Proc. of 9th IFAC Workshop on Adaptive and Learning in Control and Signal Processing, St. Petersburg, Russia CD-ROM.

Minami, A., Mizumoto, I. \& Iwai, Z. (2010). Model-based pfc design based on time-varying aspr model for anti-windup adaptive pid control, SICE Annual Conference 2010 CD-ROM: 18-21.

Mizumoto, I. \& Iwai, Z. (1996). Simplified adaptive model output following control for plants with unmodelled dynamics, Int. J. of Control 64(1): 61-80.

Ren, T.-J., Chen, T.-C. \& Chen, C.-J. (2008). Motion control for a two-wheeled vehicle using a self-tuning pid controller, Control Engineering Practice 16(3): 365-375.

Sastry, S. \& Bodson, M. (1989). Adaptive Control -Stability, Convergence, and Robustness-, Prentice Hall.

Tamura, K. \& Ohmori, H. (2007). Auto-tuning method of expanded pid control for mimo systems, Proc. of 9th IFAC Workshop on Adaptive and Learning in Control and Signal Processing, St. Petersburg, Russia CD-ROM.

Yamamoto, T. \& Shah, S. (2004). Design and experimental evaluation of multivariable self-tuning pid controller, IEE Proc. of Control Theory and Applications 151(5): 645-652.

Yu, D., Chang, T. \& Yu, D. (2007). A stable self-learning pid sontrol for multivariable time varying systems, Control Engineering Practice 15(12): 1577-1587. 


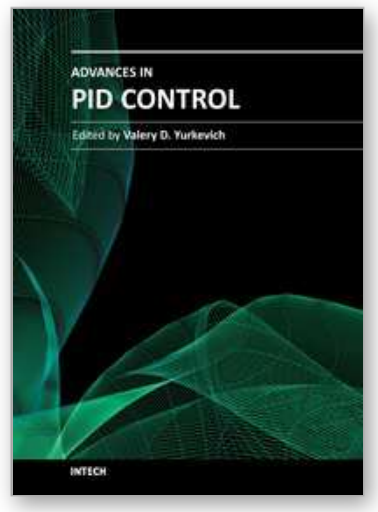

\author{
Advances in PID Control \\ Edited by Dr. Valery D. Yurkevich
}

ISBN 978-953-307-267-8

Hard cover, 274 pages

Publisher InTech

Published online 06, September, 2011

Published in print edition September, 2011

Since the foundation and up to the current state-of-the-art in control engineering, the problems of PID control steadily attract great attention of numerous researchers and remain inexhaustible source of new ideas for process of control system design and industrial applications. PID control effectiveness is usually caused by the nature of dynamical processes, conditioned that the majority of the industrial dynamical processes are well described by simple dynamic model of the first or second order. The efficacy of PID controllers vastly falls in case of complicated dynamics, nonlinearities, and varying parameters of the plant. This gives a pulse to further researches in the field of PID control. Consequently, the problems of advanced PID control system design methodologies, rules of adaptive PID control, self-tuning procedures, and particularly robustness and transient performance for nonlinear systems, still remain as the areas of the lively interests for many scientists and researchers at the present time. The recent research results presented in this book provide new ideas for improved performance of PID control applications.

\title{
How to reference
}

In order to correctly reference this scholarly work, feel free to copy and paste the following:

Ikuro Mizumoto and Zenta Iwai (2011). Adaptive PID Control System Design Based on ASPR Property of Systems, Advances in PID Control, Dr. Valery D. Yurkevich (Ed.), ISBN: 978-953-307-267-8, InTech, Available from: http://www.intechopen.com/books/advances-in-pid-control/adaptive-pid-control-system-design-based-onaspr-property-of-systems

\section{INTECH}

open science | open minds

\section{InTech Europe}

University Campus STeP Ri

Slavka Krautzeka 83/A

51000 Rijeka, Croatia

Phone: +385 (51) 770447

Fax: +385 (51) 686166

www.intechopen.com

\section{InTech China}

Unit 405, Office Block, Hotel Equatorial Shanghai

No.65, Yan An Road (West), Shanghai, 200040, China

中国上海市延安西路65号上海国际贵都大饭店办公楼405单元

Phone: +86-21-62489820

Fax: $+86-21-62489821$ 
(C) 2011 The Author(s). Licensee IntechOpen. This chapter is distributed under the terms of the Creative Commons Attribution-NonCommercialShareAlike-3.0 License, which permits use, distribution and reproduction for non-commercial purposes, provided the original is properly cited and derivative works building on this content are distributed under the same license. 